\title{
Predictors of future growth of sporadic vestibular schwannomas obtained by history and radiologic assessment of the tumor
}

\author{
Janneke C. J. M. Artz • Ferdinand C. A. Timmer • \\ Jef J. S. Mulder • Cor W. R. J. Cremers • \\ Kees Graamans
}

Received: 10 May 2008 / Accepted: 29 July 2008 / Published online: 13 August 2008

(C) The Author(s) 2008. This article is published with open access at Springerlink.com

\begin{abstract}
Management of a sporadic vestibular schwannoma (VS) is still a subject of controversy, mainly due to distinct and unpredictable growth patterns. To embark on an appropriate therapy it is necessary to dispose of a reliable prediction about tumor progression. This study aims to design a risk profile with predictors for VS growth. A total of $234 \mathrm{VS}$ patients who were managed conservatively were included. Data concerning (duration of) symptoms and localization of VS were analyzed with Cox proportional hazards regression models. Predictors for growth are unsteadiness/vertigo, no sudden onset of hearing loss and short duration of hearing loss. High-risk patients have (1) VS with an extrameatal localization, short duration of hearing loss and at least one of the two other predictors (unsteadiness/vertigo or no sudden sensorineural hearing loss) or (2) VS with an intrameatal localization and all three other predictors. Low-risk patients have (1) VS with an extrameatal component and no other predictor or (2) VS with an intrameatal localization and at most one other predictor. High-risk patients have a risk of growth of $36.9 \%$ in the first year and $64.6 \%$ in the second year. For patients with a low risk this is 2.5 and $12.7 \%$, respectively. Simple data gathered at the moment of diagnosis may provide useful information since they may lead to a risk profile for growth.
\end{abstract}

Keywords Acoustic neuroma - Growth ·

Predictors of growth $\cdot$ Risk profile $\cdot$ Symptoms .

Vestibular schwannoma

J. C. J. M. Artz · F. C. A. Timmer ( $₫)$ · J. J. S. Mulder

C. W. R. J. Cremers · K. Graamans

Department of Otorhinolaryngology, Head and Neck Surgery, Radboud University Nijmegen Medical Centre,

P. O. BOX 9101, 6500 HB Nijmegen, The Netherlands

e-mail: f.timmer@kno.umcn.nl

\section{Introduction}

The management of a unilateral sporadic vestibular schwannoma (VS) is still a subject of controversy mainly due to distinct and unpredictable growth patterns. In case of a solid VS a wait-and-scan-policy is often appropriate because these tumors in general show no growth or have a slow growth pattern, varying between 0.9 and $1.9 \mathrm{~mm}$ in maximal diameter per year [1-4]. Moreover, several studies on the growth rate of VSs, have shown besides stability even regression during conservative management [1, 5-10]. In contrast, part of the solid VSs is able to present with relatively rapid growth, which eventually might result in serious morbidity and even mortality if left untreated.

To embark on the most appropriate management of VSs it is necessary to dispose of a reliable prediction about the growth of the tumor at the moment of diagnosis.

This study is aimed to design a risk profile for VS growth based on anamnestic and radiological data gathered at the moment of diagnosis, that is (duration of) symptoms and localization of the tumor.

\section{Materials and methods}

Study group

A prospective study of patients with sporadic unilateral VSs was conducted at the department of otorhinolaryngology of the Radboud University Medical Centre Nijmegen, The Netherlands. Patients with sporadic unilateral VS were included from 1994 to 2006. The diagnosis of VS was based on characteristic symptoms, audiovestibular data and MR images. Patients who were initially managed conservatively were included. Conservative management consisted 
of a minimal follow-up of 6 months and at least two MRIscans. The treatment protocol for VS in our institute is highly individualized and flexible, particularly after informing the patient. The management is determined after considering a 10-points paradigm which includes tumor size, unfavorable health factors, invalidating symptoms, duration of symptoms, hearing level, contralateral hearing, tinnitus, balance problems, documented tumor growth and personal preferences of the patient. As a consequence, the management is tailored to the patient instead of being the result of a rigidly shaped protocol. Patients with other ear pathologies than a VS and patients with neurofibromas were not selected. Patients with cystic tumors were excluded. Cystic tumors were defined as solid tumors demonstrating cystic components filled with a fluid-like substance on the outer aspect of the tumor, as visualized with MRI.

The following patient characteristics were addressed in this study; gender, age at the moment of diagnosis, (duration of) symptoms, tumor side (left or right) and localization of the tumor. A classification was made into purely intrameatal (IAC group) or intrameatal and extrameatal [cerebellopontine angle (CPA) group]. The distinction between these two groups was made on the basis of the axial MR-images. The tumor was assigned to the IAC group when the protrusive tumor surface was less then one-third of the intrameatal tumor surface, as depicted in the axial plane with MRI. The duration of symptoms was defined as the time between the onset of first symptoms and the diagnosis.

Analysis of tumor growth

MRI was used to assess the size of the lesions. The size of the IAC tumors was taken as the maximum diameter along the length of the IAC. The diameters of the CPA tumors were measured by two different methods due to different observers. Method 1 consisted of measuring of the maximum diameter parallel to the petrous ridge in the axial plane; method 2 consisted of taking the maximum diameter parallel to the internal auditory canal in the axial plane. It is known that VS have different growth patterns. Some will show growth within the first year of observation; some will show growth after some years. Therefore, the time to first observed growth as assessed by MRI was recorded. The definition of growth was determined by the difference in tumor size of $\geq 1 \mathrm{~mm}$ in the axial plane. Growth was determined by means of independent assessments by a radiologist and an otolaryngologist.

\section{Audiometry}

\section{Pure-tone audiometry}

The audiometric assessments of the pure tone audiometry complied with the highest clinical standards. The Fletcher
Index (FI) of air conduction of the VS-ear was included. FI was defined as the mean of the thresholds at 500, 1000 and $2000 \mathrm{~Hz}$. When no threshold could be assessed in the puretone audiogram, it was noted at $130 \mathrm{~dB}$ HL.

\section{Speech audiometry}

Speech audiometry measurements with Dutch vowel-consonant-vowel words were performed up to a maximum of $125 \mathrm{~dB}$ SPL.

\section{Statistical analysis}

Statistical analysis was performed using SPSS 12.0. The occurrence of growth of the VS is dependant on the parameter "duration of follow-up". Some VS grow constantly; some VS start to grow after a period of stability and some VS stay stable. It would be inadequate to simply compare VSs in classes of "growth" and "no growth" because not all VS patients had the same period of follow-up. Therefore, the time elapsed until the first observed growth was presented in a Kaplan-Meier curve with censoring at the time of the last MRI when no growth was observed.

The association of potential risk factors with the risk of growth was examined with Cox proportional hazards regression models. Hazard rate ratios (HRR) were calculated to quantify the effect of a risk factor on growth. First, within each set of factors like localization (IAC or CPA), symptoms and duration of symptoms, the risk factors were selected using backward elimination. The selected risk factors were then combined in another backward selection procedure to yield the final model. Based on the final model regression scores can be calculated for each patient $(P<0.05)$. Patients were categorized according to quintiles of the regression score, with the lowest quintile labeled low-risk and the top quintile labeled high risk. Cumulative probability curves were constructed for patients in each of the quintiles with the use of KaplanMeier method.

\section{Results}

Study group

Prospectively 382 VS cases were considered. Conservative treatment was initially applied in 283 patients. The main reason for conservative treatment was small tumor size $(n=236)$. Some patients refused any treatment $(n=8)$, another group was managed conservatively because of comorbidity $(n=10)$ and some patients were treated conservatively because of a long history of symptoms suggesting a slow growth pattern or absence of growth $(n=26)$. A 
small group $(n=3)$ had no complaints at all and were therefore treated conservatively.

The remaining 99 patients were primarily treated by microsurgery $(n=84)$, stereotactic radiotherapy $(n=10)$ treatment or a combination of surgery and stereotactic radiotherapy $(n=5)$.

The indications for primary treatment of the VS was a large tumor size $(n=63)$, personal preference of the patient $(n=16)$, the aim of hearing preservation $(n=2)$ and the presence of debilitating audiovestibular symptoms $(n=3)$.

After exclusion of patients without a second scan $(n=38)$ and patients with cystic tumors $(n=11)$, the study group comprised 234 sporadic unilateral VS patients (Table 1). The mean age at the moment of diagnosis was 57 years (SD 11.79, range: 16-82 years), 55 years (SD 12.04, range: $16-77$ years) for men and 60 years (SD 11.10, range: $30-82$ years) for women. The distribution of the tumor localization obtained by the first MRI was as follows: 142 intrameatal and 92 intrameatal with extension into the cerebellopontine angle. The mean follow-up of these patients with conservative management was 28 months (SD: 20.89, range: 4-120 months).

\section{Tumor characteristics}

No significant difference in gender, age or side of the tumor was found between patients with and without growth of the tumor or between IAC and CPA localization. The mean maximum diameter of the IAC tumors was $9.2 \mathrm{~mm}$ (SD: 3.29 , range $2.00-14.00 \mathrm{~mm}$ ). The mean maximal diameter of the CPA tumors measured with method 1 was $12.2 \mathrm{~mm}$ (SD: 4.31, range 5.00-23.00 $\mathrm{mm}$ ). The tumors measured with method 2 had a mean maximal diameter of $17.2 \mathrm{~mm}$ (SD: 4.92, range 10.00-30.00 mm).

\section{Clinical presentation}

The symptoms reported by patients at the moment of diagnosis are shown in Table 2. The most reported symptoms at

Table 1 Patient characteristics

\begin{tabular}{|c|c|c|c|}
\hline & \multirow[t]{2}{*}{ Total } & \multicolumn{2}{|c|}{ Localization of VS } \\
\hline & & IAC & CPA \\
\hline Number of patients & 234 & 142 & 92 \\
\hline $\begin{array}{l}\text { Mean age at diagnosis } \\
\text { (years) (SD) }\end{array}$ & $57(11.79)$ & $57(11.52)$ & $59(12.16)$ \\
\hline $\begin{array}{l}\text { Mean follow-up } \\
\text { (months) (SD) }\end{array}$ & $28(20.89)$ & $31(22.50)$ & $24(17.35)$ \\
\hline Gender (male:female) & 119:115 & $71: 71$ & $48: 44$ \\
\hline Side VS (right:left) & $121: 113$ & $76: 66$ & $45: 47$ \\
\hline
\end{tabular}

$\overline{I A C}$ internal auditory canal, $C P A$ cerebellopontine angle (for definitions see "Materials and methods")
Table 2 Frequencies of symptoms

\begin{tabular}{lccc}
\hline Symptoms & $\begin{array}{l}\text { Total } \\
n=234(\%)\end{array}$ & \multicolumn{2}{l}{ Localization of VS } \\
\cline { 3 - 4 } & & $\begin{array}{l}\text { IAC } \\
n=142(\%)\end{array}$ & $\begin{array}{l}\text { CPA } \\
n=92(\%)\end{array}$ \\
\hline Hearing loss & $216(92.3)$ & $128(90.1)$ & $88(95.7)$ \\
Tinnitus & $151(64.5)$ & $93(65.5)$ & $58(63.0)$ \\
Unsteadiness/vertigo & $103(44.0)$ & $59(41.5)$ & $44(47.8)$ \\
SSHL & $31(13.2)$ & $20(14.1)$ & $11(12.0)$ \\
Aural fullness & $27(11.5)$ & $19(13.4)$ & $8(8.7)$ \\
Otalgia & $10(4.3)$ & $5(3.5)$ & $5(5.4)$ \\
Headache & $8(3.4)$ & $3(2.1)$ & $5(5.4)$ \\
\hline
\end{tabular}

SSHL sudden sensorineural hearing loss

their first visit to the hospital were hearing loss, tinnitus and unsteadiness/vertigo. Also sudden sensorineural hearing loss (SSHL) and aural fullness were a rather common presenting symptom in 13.2 and $11.5 \%$ of the patients, respectively. The mean duration of all symptoms was 60 months (SD 80.42, range 1-480 months). In the total patient population sample hearing loss had the longest duration amongst other symptoms (Table 3).

In the study group no other otologic disorders were present.

\section{Audiometry}

Pure tone audiometry data of 231 patients was available. Data of the audiometry results for the VS-ear are presented in Table 4. The mean FI was $38.9 \mathrm{~dB}$ HL (SD $25.98 \mathrm{~dB}$ HL). In six patients total deafness of the VS-ear was found (FI $\geq 130 \mathrm{~dB} \mathrm{HL}$ ). Speech recognition audiometry data of 211 patients were available. The mean Pbmax was $70.0 \%$ (SD 29.85) (Table 4).

\section{Risk of growth}

The risk of growth in the first year after diagnosis regarding the whole study population sample is $17 \%$ and is increasing during follow-up. Within 2 years the risk increases to $38 \%$ and within 5 years it increases to 58\%. Tumors with an initial IAC localization have a $10 \%$ risk of growth within 1 year compared to $28 \%$ for tumors with an initial CPA localization. Within 3 years this risk is $43 \%$ compared to $64 \%$ for intrameatal and extrameatal tumors, respectively. Within 5 years the risk seems stable for patients with intrameatal tumors (49\%) and seems to increase for patients with extrameatal tumors $(73 \%)$. This results in a HRR of 2.0 for growth $(P<0.05)$ for patients with a VS with extension into the cerebellopontine angle compared to patients with a VS which is limited to the internal auditory canal (Fig. 1). 
Table 3 Mean duration of symptoms

\begin{tabular}{llll}
\hline Symptoms & $\begin{array}{l}\text { Total } \\
\text { Months (SD) }\end{array}$ & \multicolumn{2}{l}{ Localization of VS } \\
\cline { 3 - 4 } & & $\begin{array}{l}\text { IAC } \\
\text { Months (SD) }\end{array}$ & $\begin{array}{l}\text { CPA } \\
\text { Months (SD) }\end{array}$ \\
\hline Hearing loss & $55(76.9)$ & $48(74.84)$ & $65(79.15)$ \\
Tinnitus & $37(56.80)$ & $31(51.02)$ & $47(64.32)$ \\
Unsteadiness/vertigo & $39(68.43)$ & $38(65.32)$ & $40(37.65)$ \\
SSHL & $28(58.34)$ & $17(41.84)$ & $41(73.90)$ \\
Aural fullness & $21(25.79)$ & $14(14.94)$ & $50(41.68)$ \\
Otalgia & $7.0(6.61)$ & a & a \\
Headache & $32(48.98)$ & a & a \\
\hline
\end{tabular}

SSHL sudden sensorineural hearing loss

a Patient number $\leq 5$

Table 4 Audiometry data of the ear with VS

\begin{tabular}{llll}
\hline & Total & \multicolumn{2}{l}{ Localization of VS } \\
\cline { 3 - 4 } & Mean (SD) & $\begin{array}{l}\text { IAC } \\
\text { Mean (SD) }\end{array}$ & $\begin{array}{l}\text { CPA } \\
\text { Mean (SD) }\end{array}$ \\
\hline FI (dB HL) & $38.9(25.98)$ & $36.9(26.18)$ & $42.0(25.50)$ \\
Pbmax (\%) & $70.0(29.85)$ & $73.3(28.61)$ & $65.0(31.15)$ \\
\hline
\end{tabular}

FI Fletcher Index, Pbmax maximum speech recognition score

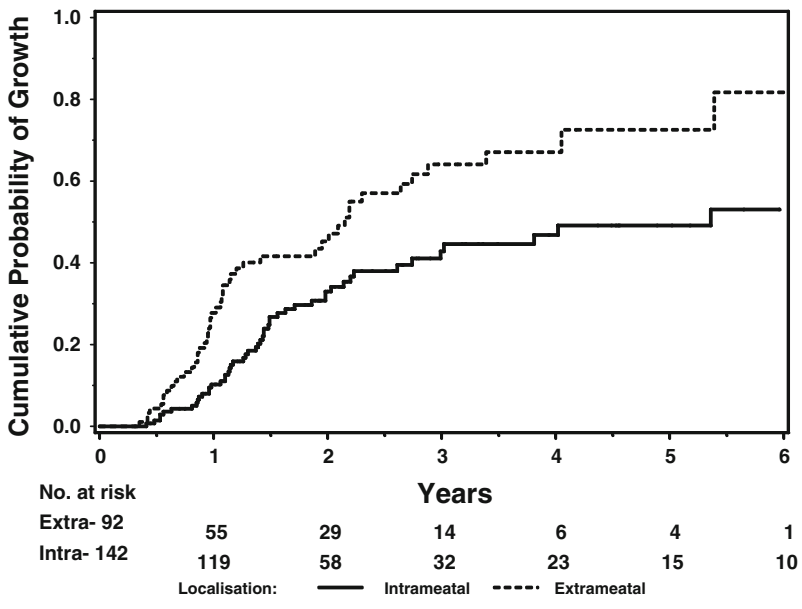

Fig. 1 Risk of growth for VS with intrameatal versus extrameatal localization at the moment of diagnosis

\section{Potential risk factors and predictive model}

The following potential risk factors are retained as predictors of growth in backward elimination models: an extrameatal localization of VS and among symptoms, tinnitus, unsteadiness/vertigo, no SSHL and a short duration of hearing loss. This short duration is defined as a hearing loss of just 1-24 months.

The final model is based on population sample of 224 patients; in 10 patients no information about duration of symptoms was reported. The following predictors of growth are included in the model 1: extrameatal localization (HRR 2.3, $P<0.0001$ ), 2: no SSHL (HRR 0.5, $P<0.05), 3$ : unsteadiness/vertigo (HRR 2.0, $P<0.001$ ) and 4: short duration of hearing loss (1-24 months) (HRR $3.1(P<0.0001)$. The symptom tinnitus as predictor of growth had no contributory effect to the model. Based on this final model 16 classes of patients with different risk profiles can be defined. We categorized the patients according to quintiles of the regression score. The cumulative probability of growth for each quintile is obtained with the Kaplan-Meier method. The following risk profiles were defined (1) high-risk $(n=43)$, (2) intermediate risk $(n=139)$ and (3) low-risk $(n=42)$. The patients with a high and low-risk for growth of VS are shown in Table 5.

For patients with a low-risk profile the risk of growth of a VS is $2.5 \%$ in the first year and $12.7 \%$ within 2 years. For patients with a high-risk profile the risk of growth of a VS in the first year is 36.9 and $64.6 \%$ within 2 years (Fig. 2).

\section{Discussion}

Vestibular schwannomas are mostly slow growing tumors. Conservative management with regularly MRI-scanning is an appropriate approach because any type of therapy may result into (increased) hearing loss or facial nerve deficits. However, some VS present with rapid growth that may lead to serious morbidity and even mortality if left untreated. Preferably, the growth behavior of VS should be predictable at the moment of diagnosis to allow the best strategy. Throughout the years, several studies attempted to identify growth predictors of VSs. Due to different research hypotheses and protocols the results of these studies are hardly comparable. The size of the tumor at the moment of diagnosis has been proposed as an indicator for growth. Fucci et al. [11] reported a significantly increased growth rate of tumors with a maximal diameter larger than $20 \mathrm{~mm}$ at presentation. Some studies found that tumor growth in the first year could be indicative for future growth $[12,13]$. Furthermore the symptoms of the patients have been considered as possible predictors of growth. Tschudi et al. [13] found that patients with progressive hearing loss as a first symptom had a significantly lower tumor growth than those presenting with tinnitus, sudden hearing loss and vertigo. Other studies, however, revealed no significant differences in age, gender, tumor laterality, initial symptoms, duration of symptoms or initial tumor size between patients with growing tumors and non-growing tumors [7, 11, 12, 14]. A possible predictor of growth may be the duration of symptoms. One study reported that patients with a short duration of symptoms had tumors that significantly grew faster [15]. Our study demonstrated that the symptom hearing loss was 
Table 5 Risk profile of patients with VS

The predictors for growth are extrameatal localization and among symptoms, short duration of hearing loss (1-24 months), unsteadiness/vertigo, no SSHL

\begin{tabular}{lc}
\hline High risk & Low risk \\
\hline $\begin{array}{l}\text { 1. VS with an extrameatal component and } \\
\text { Short duration of hearing loss and } \\
\text { At least one of the other two predictors } \\
\text { (unsteadiness/vertigo or no SSHL) }\end{array}$ & $\begin{array}{c}\text { 1. VS with an extrameatal component and } \\
\text { No other predictor }\end{array}$ \\
$\begin{array}{l}\text { 2. VS with intrameatal localization and } \\
\text { All three other predictors }\end{array}$ & $\begin{array}{c}\text { 2. VS with an intrameatal localization and } \\
\text { At most one other predictor }\end{array}$ \\
\hline
\end{tabular}

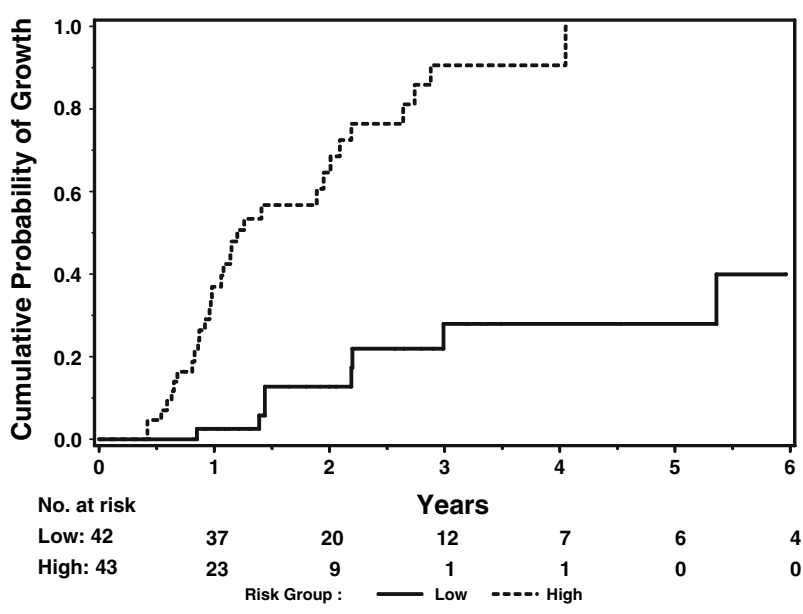

Fig. 2 Patients with high and low-risk of growth of VS. High-risk and low-risk groups are mentioned in Table 5

present in $92.3 \%$ of the patient population sample and that short duration of hearing loss was indeed a predictor of growth with a hazard risk of $3.1(P<0.0001)$.

The search for predictors of growth should result in a manageable profile which could be implied in practice. Such a profile can be performed by using statistical tests like regression or multivariate analysis. Beenstock [16] used a multivariate analysis to investigate predictors for VS growth. However, this study was based on two independent sets of previous data with a small number of patients ( $n=51$ and $n=69)$. Age, side of the tumor and reporting symptoms seemed to be key variables in predicting stability or growth of VS [16]. If reporting symptoms included vertigo and unsteadiness in tumor growth was more pronounced. This could be confirmed in our study: the hazard risk for growth for the symptom unsteadiness/vertigo was 2.0. Beenstock [16] found that probability of stability seemed to be independent of gender, tinnitus and hearing loss. This is also in accordance with the results found in our study except for tinnitus which was retained as a predictor for growth. In this study side of the tumor was not indicative as a potential risk factor for growth. Beenstock [16] reported that left side of the tumor was an indicator for growth of VS. Furthermore in one data set VS growth varied significantly and inversely with age.

This study was able to present a risk profile of VS growth concerning initial symptoms and localization of VS.
In contrast to results of earlier studies, we demonstrated that symptoms reported by patients at the moment of diagnosis seem to be of great value in predicting growth behavior of VS. More attention need to be given to initial symptoms such as unsteadiness or vertigo, tinnitus, short duration of hearing loss or the absence of SSHL. This in combination with the localization of the VS as seen on the first MRI might be of importance considering treatment options for VS. To our knowledge this is the first study that presents a risk profile for VS growth based on information gathered at the moment of diagnosis.

The statistical grouping of patients in three risk groups and comparing the high and low-risk groups eliminated a considerable amount of patients from the study. However, this grouping was essential in order to distinguish relevant differences. The outline of the statistical analysis was shaped in such a way that the effects of the various determinants could be visualized.

Studies concerning the prediction of stability or growth of VSs should ideally be done with random patient selection. The non-random selection method might have resulted in overstatement of stability of the VS and understatement of initial symptoms. In clinical practice, however, a random selection of patients with VS is not feasible, because some VSs have such large size that surgery instead of conservative management is the treatment of first choice. The differences between the treated and non-treated groups of patients were quite variable and not uniformly categorized. This makes that some degree of similarity between those two groups is certainly present. As a result the outcomes of this longitudinal study should be considered as being representative or at least indicative for the whole VS population.

\section{Conclusion}

This study presents a risk profile for VS growth based on symptoms and localization of the tumor in a non-randomized study group managed by a "wait and scan" policy. Patients with a high-risk profile have a risk of growth of $36.9 \%$ in the first year and $64.6 \%$ within 2 years. To this risk profile belong patients with (1) VS with an extrameatal component and short duration of hearing loss and at least one of the two predictors (unsteadiness/vertigo or no 
SSHL), (2) VS with intrameatal localization and all three other predictors (short duration of hearing loss, unsteadiness/vertigo, no SSHL). For patients with a low-risk profile the risk of growth in the first year is 2.5 and $12.7 \%$ within 2 years. These patients have (1) VS with an extrameatal component and no other predictor or (2) VS with an intrameatal localization and at most one other predictor.

The outcomes of this study cannot directly be generalized for the whole VS population. Nevertheless, they are indicative for the behavior of these tumors.

Acknowledgments The authors thank Mrs. S. Peer for statistical analysis.

Open Access This article is distributed under the terms of the Creative Commons Attribution Noncommercial License which permits any noncommercial use, distribution, and reproduction in any medium, provided the original author(s) and source are credited.

\section{References}

1. Rosenberg SI (2000) Natural history of acoustic neuromas. Laryngoscope 110(4):497-508. doi:10.1097/00005537-200004000-00002

2. Smouha EE, Yoo M, Mohr K, Davis RP (2005) Conservative management of acoustic neuroma: a meta-analysis and proposed treatment algorithm. Laryngoscope 115(3):450-454. doi:10.1097/ 00005537-200503000-00011

3. Warrick P, Bance M, Rutka J (1999) The risk of hearing loss in nongrowing, conservatively managed acoustic neuromas. Am J Otol 20(6):758-762

4. Yoshimoto Y (2005) Natural history of vestibular schwannoma. Nippon Rinsho 63(Suppl 9):321-323

5. Bozorg GA, Kalamarides M, Ferrary E et al (2005) Conservative management versus surgery for small vestibular schwannomas. Acta Otolaryngol 125(10):1063-1068. doi:10.1080/000164805 10038013
6. Charabi S, Tos M, Thomsen J, Charabi B, Mantoni M (2000) Vestibular schwannoma growth: the continuing controversy. Laryngoscope 110(10 Pt 1):1720-1725. doi:10.1097/00005537200010000-00030

7. Flint D, Fagan P, Panarese A (2005) Conservative management of sporadic unilateral acoustic neuromas. J Laryngol Otol 119(6):424-428. doi:10.1258/0022215054273089

8. Massick DD, Welling DB, Dodson EE et al (2000) Tumor growth and audiometric change in vestibular schwannomas managed conservatively. Laryngoscope 110(11):1843-1849. doi:10.1097/ 00005537-200011000-00015

9. Raut VV, Walsh RM, Bath AP et al (2004) Conservative management of vestibular schwannomas - second review of a prospective longitudinal study. Clin Otolaryngol Allied Sci 29(5):505-514. doi:10.1111/j.1365-2273.2004.00852.x

10. Stipkovits EM, Graamans K, Van Dijk JE (2001) Vestibular schwannoma: negative growth and audiovestibular features. Eur Arch Otorhinolaryngol 258(9):467-471. doi:10.1007/s004050 100369

11. Fucci MJ, Buchman CA, Brackmann DE, Berliner KI (1999) Acoustic tumor growth: implications for treatment choices. Am J Otol 20(4):495-499

12. Bederson JB, von Ammon K, Wichmann WW, Yasargil MG (1991) Conservative treatment of patients with acoustic tumors. Neurosurgery 28(5):646-650. doi:10.1097/00006123-19910 5000-00002

13. Tschudi DC, Linder TE, Fisch U (2000) Conservative management of unilateral acoustic neuromas. Am J Otol 21(5):722-728

14. Herwadker A, Vokurka EA, Evans DG, Ramsden RT, Jackson A (2005) Size and growth rate of sporadic vestibular schwannoma: predictive value of information available at presentation. Otol Neurotol 26(1):86-92. doi:10.1097/00129492-200501000-00015

15. Charabi S, Thomsen J, Mantoni M et al (1995) Acoustic neuroma (vestibular schwannoma): growth and surgical and nonsurgical consequences of the wait-and-see policy. Otolaryngol Head Neck Surg 113(1):5-14. doi:10.1016/S0194-5998(95)70138-9

16. Beenstock M (2002) Predicting the stability and growth of acoustic neuromas. Otol Neurotol 23(4):542-549. doi:10.1097/ 00129492-200207000-00025 